\title{
LA CIUDAD Y SUS MAPAS: REPRESENTACIONES CARTOGRÁFICAS DE ÁMSTERDAM EN EL ARTE CONCEPTUAL
}

Esmeralda Gómez Galera 


\section{THE CITY AND ITS MAPS: CARTOGRAPHIC REPRESENTATIONS OF AMSTERDAM IN CONCEPTUALART}

\section{RESUMEN}

Este escrito propone una reflexión sobre el mapa como mecanismo de representación del territorio y como dispositivo poético apropiable por las prácticas artísticas. Con tal fin, realiza un recorrido por la obra de tres artistas conceptuales que desarrollaron parte de su producción en Ámsterdam durante las décadas de los sesenta y setenta. En este contexto, Sol LeWitt, Stanley Brouwn y Jan Dibbets, reunidos en torno a la galería Art \& Project, desarrollaron representaciones fragmentarias y experienciales de la ciudad a través del uso de mapas.

Palabras clave: cartografía, representación, ciudad, arte conceptual, Ámsterdam.

\section{ABSTRACT}

This paper reflects on maps both as an instrument for the representation of territory and as a poetic instrument which is appropriable by artistic practices. To do so it analyzes the work of three conceptual artists who developed part of their production in Amsterdam during the sixties and seventies. In this context, Sol LeWitt, Stanley Brouwn and Jan Dibbets, gathered around the Art \& Project gallery, developed fragmentary and experiential representations of the city through the use of maps.

Keywords: Cartography, representation, city, conceptual art, Amsterdam.

\section{AUTORES}

\section{Esmeralda Gómez Galera}

Artista e investigadora.

Bellas Artes.

Universidad de Castilla-La Mancha.

Máster de investigación en Prácticas artísticas y visuales.

Universidad de Castilla-La Mancha

Correo electrónico: esmeralda.gomez.galera@gmail.com 


\section{EL MAPA-FRAGMENTO}

El mapa sustituye torpemente al territorio. Entre la representación cartográfica y la realidad hay un abismo insalvable y misterioso, una distancia que permite al mapa abarcar una ciudad entera con vista de pájaro. En el esfuerzo de los cartógrafos confluyen ciertas dosis de creatividad con una pretensión de precisión y de totalidad. Como resultado, el mapa nos muestra una determinada visión de la realidad que no está exenta de ideología. La pretensión, siempre frustrada, de abarcar representacionalmente lo real conlleva también un aplanamiento de sus complejidades, cuya naturaleza vendrá determinada por posiciones ideológicas dominantes o imaginarios hegemónicos. Por supuesto, la representación cartográfica produce olvidos y genera siempre un afuera: ¿Qué no puede contar el mapa? ¿Qué queda más allá de sus márgenes? ¿Qué elementos son simplemente obviados en la piel de su superficie abstracta?

Desde el plano literario, tanto Lewis Carroll como Jorge Luis Borges reflexionaron lúcidamente sobre las paradojas de la compleja relación entre cartografía y territorio, entre la siempre insuficiente representación de lo real y la realidad misma. Ambos fantasean con la idea de un mapa total y, por ello mismo, inútil o imposible de desplegar. Se trata de un mapa perfecto y fiel, que no escala la realidad, sino que representa el territorio en su tamaño original sin obviar ningún detalle. Nos topamos aquí, bruscamente, con el límite de la representación cartográfica. En palabras de Mein Herr, el personaje de Lewis Carroll:

"Nosotros superamos muy pronto las seis yardas por milla. Luego probamos con cien yardas por milla. ¡Y finalmente llegó la idea más fabulosa de todas! ¡Realizamos un mapa del país, a escala de una milla por milla!"

“¿Lo habéis utilizado mucho?” pregunté.
"Nunca ha sido desplegado todavía" dijo Mein Herr, "los granjeros se opusieron. Dijeron que cubriría completamente el país iy no dejaría pasar la luz del sol! Así que ahora utilizamos el mismo país como su propio mapa, y te aseguro que funciona casi tan bien". (2015, p.183. Trad. propia).

De manera inevitable, el resultado de esta creciente fidelidad del mapa es una renuncia a la misma idea de representación. Por su parte, en el breve cuento de Borges "Del rigor en la ciencia", el mapa es igualmente inservible debido a su extrema fidelidad con el territorio representado. El imperio imaginado por Borges perfecciona hasta tal punto la ciencia de la cartografía, que logra elaborar un mapa a escala 1:1 del imperio. No hay, por tanto, necesidad alguna de representar, pues en los dos ejemplos citados la presencia el territorio acaba imponiéndose sobre la representación cartográfica, por más fidedigna que esta sea. Tanto el mapa de Carroll como el de Borges quedan reducidos a su incapacidad de sustitución simbólica del territorio que intentan representar:

Menos adictas al estudio de la cartografía, las generaciones siguientes entendieron que ese dilatado mapa era inútil y no sin impiedad lo entregaron a las inclemencias del sol y de los inviernos. En los desiertos del Oeste perduran despedazadas ruinas del Mapa, habitadas por animales y por mendigos [...]. (Borges, 1974, p.847).

Así es como el mapa, en su imposible y frustrado afán de perfección, se topa con su propio límite y termina siendo una ruina habitable, una piel fragmentada. No obstante, el mapa-fragmento no es el resultado necesario de un devenir-ruina motivado por la imposibilidad de abarcar representacionalmente lo real. También puede el mapa ser concebido inicialmente como fragmento, esto es, nacer de 
una renuncia a la pretensión de totalidad y fidelidad representacional en favor de aspiraciones más discretas. Existe aquí un contraste importante con respecto a los mapas descritos por Carroll y Borges: una apuesta por la parcialidad o fragmentariedad en los mecanismos de representación cartográfica. De este modo, el mapa dejaría de ser, en palabras de Graciela Speranza, un "coloso mitológico" (2012, p.23) que carga con la información del mundo y tiene una vista cenital del mismo. El coloso es un gigante de dimensiones y fuerzas sobrehumanas que, desde su inmensa altura, puede efectuar una apropiación visual del territorio. Por ello mismo, encarna la fantasía panóptica y omnisciente que consiste en verlo todo. La representación está siempre ligada a un punto de vista, normalmente determinado por una cuestión de poder, de dominio.

No obstante, el mapa-fragmento al cual me refiero muestra su afinidad con otra criatura propia de la mitología griega. La ninfa es una deidad menor cuya existencia está estrechamente ligada a un lugar concreto de la geografía: río, montaña, arboleda, cascada, pradera. Esto impide la vista de pájaro a la cual se aproxima peligrosamente el coloso, proporcionando, en cambio, una perspectiva parcial y situada, nunca desligada de la experiencia del fragmento. El mapafragmento depone aquí su pretensión de totalidad representacional con la intención de contar historias situadas, de dar cuenta de la experiencia.

\section{DE PARÍS AÁMSTERDAM: EL MAPA-FRAGMENTO SITUACIONISTA}

Fuera del mundo literario, también numerosos artistas han reflexionado sobre las paradojas de la representación cartográfica y empleado mapas con distintos propósitos: "desnaturalizar los órdenes instituidos, interrogar las identidades territoriales, tender pasajes en fronteras infranqueables, conjeturar otros mundos posibles y trazar recorridos imaginarios" (Speranza,
2012, p.23). Por supuesto, también se han ideado mapas con otros fines, incluso aquellos que parecen entrar en contradicción directa con su función original... ¿Un mapa para la desorientación personal?

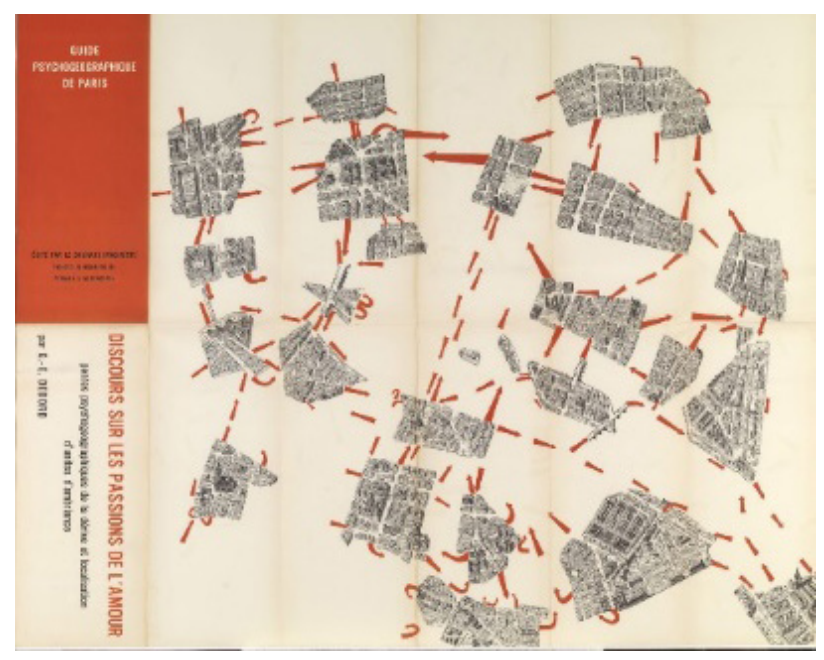

Imagen 1. Guy Debord. Guide Psychogeographique de Paris, 1957.

Tal es el caso de Guide Psychogeographique de Paris, realizada por Guy Debord en 1957. Se trata del primer mapa psicogeográfico situacionista $\mathrm{y}$, como tal, la fidelidad en la representación cartográfica de la urbe es sustituida por el intento de representar las sensaciones producidas por la psicogeografía en los trayectos urbanos. Al contrario que el mapa descrito por Borges, mapa-totalidad que deviene ruina, el de Debord es un mapa-fragmento desde su propia concepción. En el vacío blanco, flotan como icebergs diversos fragmentos de París desconectados entre sí, pero, al mismo tiempo, susceptibles de ser puestos nuevamente en conexión por medio de las posibles trayectorias sugeridas mediante flechas rojas multidireccionales. Tanto por su fragmentación estratégica como por el dinamismo que las flechas 
sugieren, la ciudad parece estar en fuga, atrapada en una tensión irresoluble entre la integración y la desintegración total. Esta es impedida por la potencialidad que trae consigo el caminante a la deriva, dispuesto a perderse y a dotar de sentido parcial a un París al borde del desmembramiento.

Sin duda, es significativa la presencia del vacío en la Guide Psychogeographique de Paris. En lugar de imposibilidad o límite representacional, el vacío en este mapa es recordatorio de nuestra limitada experiencia de la urbe contemporánea, con base en usos cotidianos de la misma. El hábito nos rapta a la hora de habitar la ciudad y, de acuerdo a las teorías situacionistas, el principio de la psicogeografía unido a la experiencia lúdica de la deriva puede rescatarnos de ese rapto. Los miembros de la Internacional Situacionista hicieron uso recurrente de mapas fragmentados para dar cuenta de sus experiencias urbanas ${ }^{1}$, sentando un antecedente importante para los usos posteriores del mapa en las prácticas artísticas de los sesenta y setenta.

Experiencia-fragmento de la ciudad, mapa-ninfa que la representa contando una pequeña historia de la parcialidad, la experiencia situacionista proporciona numerosos ejemplos al respecto. Sin embargo, en esta ocasión no es París, sino Ámsterdam, la ciudad que me gustaría analizar en relación a los mapas que la cuentan. El caso de Ámsterdam es destacable, pues la imagen de la ciudad, con su peculiar trazado urbano de calles y canales, ha desatado fascinación en numerosos artistas que han elaborado muy distintas representaciones cartográficas de la misma.
Lo que estos mapas tienen en común es su carácter parcial y fragmentario, su intención de acoger pequeñas narrativas. Me detendré fundamentalmente en el trabajo de tres artistas conceptuales que desarrollaron su actividad creativa en la capital neerlandesa durante las décadas de los sesenta y setenta: Sol LeWitt, Stanley Brouwn y Jan Dibbets.

\section{REPRESENTACIONES CARTOGRÁFICAS DE ÁMSTERDAM}

\subsection{La ciudad recortada: Sol LeWitt}

Sol LeWitt llegó a Ámsterdam en los setenta, como muchos otros artistas de su generación. Aunque la experiencia revolucionaria de mediados de la década anterior había fracasado, se respiraba en la ciudad un ambiente de libertad y desarrollo cultural que atrajo a un creciente grupo de artistas, cuya obra se caracterizó por su afinidad con las prácticas conceptuales ${ }^{2}$, una visión expandida de la escultura y gran atención al contexto de la ciudad y su especificidad urbana:

Artistas de todo el mundo vinieron a Ámsterdam en las décadas de 1960 y 1970, atraídos por museos innovadores, un prometedor sistema de galerías de arte, políticas sociales progresistas, y la ciudad misma, cuya historia había sido moldeada por sucesivas oleadas de emigración e inmigración. (Cherix, 2009. Trad. propia).

Por citar algunos ejemplos significativos que aportan pistas sobre el clima cultural del momento, la galería Art \& Project estaba abierta desde septiembre

$1 \quad$ Algunos ejemplos destacables son los collages de Gilles Ivain, la novela gráfica situacionista The Leaning Tower of Venice (1957) de Raph Rumney o el libro Mémories (1959), fruto de una colaboración entre Guy Debord y Asger Jorn.

$2 \quad$ La exposición In \& Out of Amsterdam: travels in conceptual art exploró a través del trabajo de Bas Jan Ader, Lawrence Weiner Stanley Brouwn o Allen Ruppersberg, entre otros; el desarrollo de una incipiente escena artística conceptual en la capital neerlandesa, ligada sobre todo a la actividad de la galería Art \& Project. Comisariada por Christophe Cherix, la exposición tuvo lugar del 19 de julio al 5 de octubre de 2009 en el MoMa. 
de 1968, en 1971 el Stedelijk Museum organiza la primera exposición de sus Conceptual Series, en las cuales se incluirán muestras individuales de Baldessari, Brouwn o el propio Sol LeWitt; y en 1975 Ulises Carrión abriría su espacio Other Books and So. En este contexto, Sol LeWitt producirá para la galería Art \& Project una serie de trabajos basados en intervenciones sobre mapas comerciales o turísticos de Ámsterdam.

Las obras resultantes se caracterizan por desplegar una visión fragmentaria de la ciudad, si bien la fragmentación ocurre en dos posibles direcciones: como vacío o como lleno. En el primer caso, fragmentación-vacío, formas geométricas irregulares son extraídas del mapa, dejando tras de sí un espacio en blanco, como una amnesia caprichosa en el plano de la ciudad. Lo que determina la forma del área extraída son los puntos de referencia del artista: lugares habitados o trayectos recurrentes, los cuales pueden conectarse entre sí para abarcar áreas completas. Un ejemplo es Map of Amsterdam with the area between the Dam, Art and Project, Utrechtse-Brug, Zeeburgerstraat and Achtergracht removed (1976). En el segundo caso mencionado, fragmentación-lleno, Sol LeWitt selecciona fragmentos de la ciudad con base en puntos de referencia, conserva esas áreas y retira el resto del mapa. Area of Amsterdam Between Leidseplein, Jan Dibbet's House, and Kunstijsbaan Japeden (1976) muestra un triángulo irregular de Ámsterdam, resultado de la unión de tres puntos geográficos de la ciudad.

A través de esta técnica de recorte o cut-out, el artista interviene sobre el mapa turístico para generar otro mapa, el que cuenta la historia de una ciudad habitada, de ciertos usos rutinarios de la misma. Lugares recurrentes, como la casa de Dibbets o el Museo Stedelijk, motivan también recorridos frecuentes. Si bien dichos puntos remiten a las vivencias urbanas

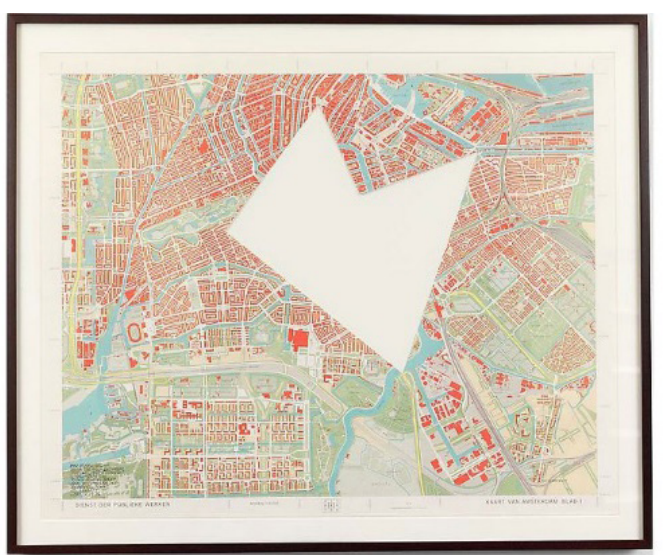

Imagen 2. Sol Lewitt. Map of Amsterdam with the area between the Dam, Art and Project, UtrechtseBrug, Zeeburgerstraat and Achtergracht removed. 1976.

de LeWitt, la razón por la que están contenidos en los títulos es doble. Por un lado, la imagen-ciudad es inevitablemente parcial. Por otro, debido a su autorreferencialidad, los títulos se convierten también en una suerte de instrucciones que cualquiera puede ejecutar para obtener su propio mapa. Con posterioridad a la realización de esta serie de mapas, LeWitt realizará trabajos similares en otras ciudades como Nueva York, Chicago o Florida:

Las piezas de Ámsterdam parecen ser sus primeras obras creadas a partir de mapas comerciales de ciudades, guías esenciales para un turista en una ciudad definida por calles curvadas y canales. Alterados por LeWitt, sugieren su afinidad y compromiso con un lugar que resultó ser fértil para su desarrollo artístico. (Cherix, 2009, p.100. Trad. propia).

En estas primeras experimentaciones con mapas, la ciudad de Ámsterdam también es sometida otro tipo de extracciones. Por ejemplo, en uno de los 
trabajos de LeWitt los numerosos parques de la ciudad han desaparecido. Pero sin duda el ejercicio de extracción más impresionante es el del río Amstel. El resultado es una ciudad completamente distinta que parece haberse derramado por los bordes y cuya estructura radial de canales y puentes aparece ahora como una decisión caprichosa sin el río que ha esculpido la fisionomía de la ciudad, que ha sido la razón de su trazado urbanístico. Continuando el juego dialéctico fragmento-vacío, fragmento-lleno, varias décadas después de la serie de Sol LeWitt, el artista argentino Jorge Machi realizó el ejercicio opuesto: extrajo calles y manzanas de la ciudad para dejar de ella tan solo las venas azules por las que transcurre el agua. Dos mapas cuentan la misma ciudad en idiomas distintos: el lleno y el vacío. Una Ámsterdam solo río, inundada en sí misma y una Ámsterdam seca, pacientemente disecada.

\section{2. La ciudad y sus huellas: Stanley Brouwn}

Acorde a la información que proporcionan los escasos datos biográficos disponibles, Stanley Brouwn llegó a Ámsterdam en 1957, tras mudarse de su país natal, Surinam, aún colonia de los Países Bajos ${ }^{3}$. Su actividad artística en este período se basó en contactos con desconocidos, en un intento de problematizar el concepto de autoría artística. En 1960, Brouwn distribuyó hojas de papel en las calles de Ámsterdam con la intención de registrar las huellas de viandantes o ciclistas. Esas huellas registran el movimiento y el tiempo en Ámsterdam, la inmensa coreografía urbana que nos hace transitar por un lugar de la ciudad en un instante concreto.

En 1961 el artista comenzó a trabajar en el proyecto titulado This way Brouwn, el cual reúne un conjunto

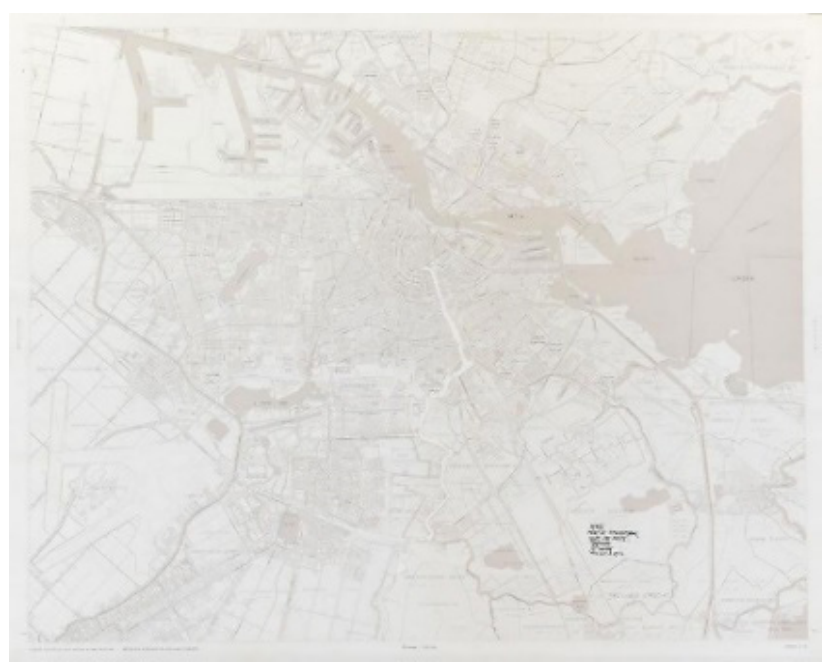

Imagen 3. Sol LeWitt. Map of Amsterdam with the Amstel (river) Removed, 1976.

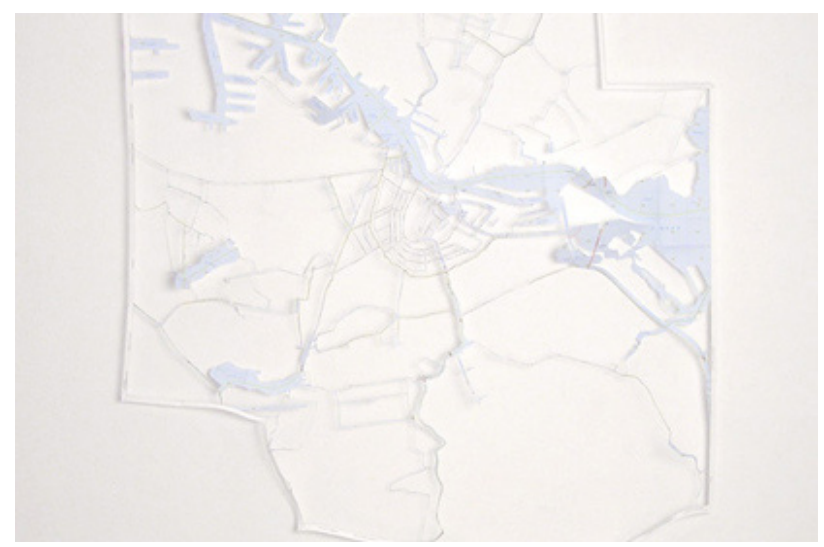

Imagen 4. Jorge Macchi. Ámsterdam, 2004. 
de representaciones cartográficas de Ámsterdam creadas a partir del encuentro con peatones en el espacio público. En dichos encuentros, Brouwn solicitó indicaciones para llegar a ciertos lugares de la ciudad. Normalmente, las explicaciones de sus interlocutores van acompañadas de un pequeño croquis o mapa mínimo en una hoja de papel facilitada por el propio artista. Sobre esta cartografía improvisada quedan estampadas las palabras que dan título a la obra: This way brouwn. En ocasiones, solo el título es estampado en un papel en blanco cuando los interlocutores del artista no han dibujado nada en él. La firma-sello y la participación de desconocidos permiten a Brouwn difuminar la figura del autor y adoptar cierta indiferencia estética, pues según sus propias palabras: "una obra de la serie this way brouwn se produce en el tiempo en que el peatón tarda en dar la explicación. No hay dudas ni correcciones. El resultado se muestra tal cual. No hay this way brouwns buenos y malos" (Brouwn, 1971).

En esta serie, las representaciones de la ciudad van más allá de la materialidad de los mapas, son también una elocución-relato. No obstante, cuando los encuentros se materializan en la superficie del papel, nos encontramos ante un mapa muy distinto al modelo del mapa turístico oficial, incluso si conduce a lugares igualmente emblemáticos de la ciudad. En una de las piezas de la serie, Brouwn solicita direcciones para llegar a la Damplatz, lugar central de Ámsterdam que acoge el Palacio Real. El resultado de este encuentro es un mapa no estático, sino direccional, una representación parcial en la cual el espacio intermedio entre el punto de partida y el de llegada se reviste de consistencia. La plaza es representada esquemáticamente como un cuadrado. En torno a ella se distribuyen algunos edificios dibujados como formas rectangulares y un círculo, probablemente en representación del Monumento Nacional, circular en su planta. Estas cartografías anónimas no están dominadas por una obsesión de precisión ni de totalidad, en ellas tanto la representación del espacio como la escala son completamente relativas y se encuentran ligadas a la experiencia urbana del peatón.

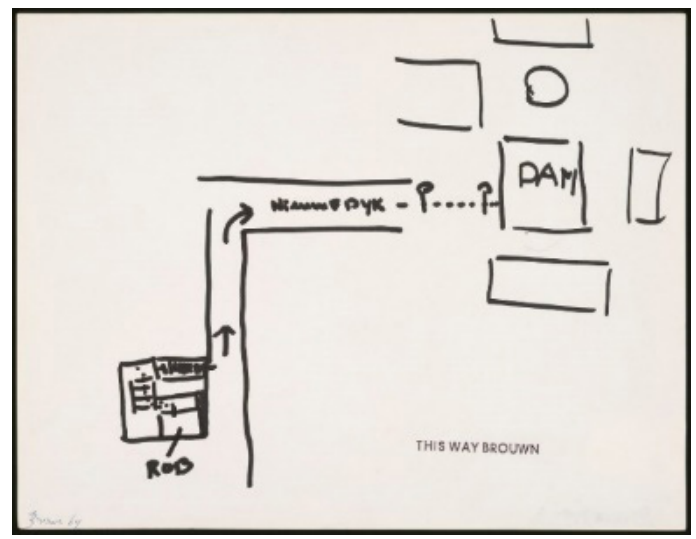

Imagen 5. Stanley Brouwn. This way Brouwn, 1964.

La fascinación de Brouwn por el acto de caminar lo condujo, en las décadas siguientes, a contar sus pasos en diferentes países, a medir obsesivamente distancias en pies o pasos como unidades de medida. Esta fascinación se manifiesta también en su propuesta para el número 11 del boletín de la galería Art \& Project, publicado en 1969. En él, Brouwn simplemente escribió unas escuetas instrucciones: "Walk during a few moments very consciously in a certain direction; simultaneously an infinite number of living creatures in the universe are moving in an infinite number of directions" (Brouwn, 1969).

\section{CARTOGRAFÍAR LOS DETALLES: JAN DIBBETS}

Sol LeWitt y Stanley Brouwn no fueron los únicos artistas con base temporal o permanente en Ámsterdam que trabajaron con mapas de la ciudad y 
que mostraron, en sus proyectos, nuevas visiones de la misma. También el artista conceptual holandés Jan Dibbets prestó atención a la representación cartográfica de la urbe, a la cual llegó después de su estancia en la St. Martin School de Londres en 1967. En sus diferentes propuestas, Dibbets captó magistralmente los pequeños, casi insignificantes, detalles de la vida en la ciudad, el transcurso temporal y las modificaciones espaciales de la misma. En la cotidianeidad, en los acontecimientos que a menudo pasan desapercibidos en la vida urbana, encontró el artista un inagotable campo de exploración conceptual. Un buen ejemplo de ello es The Shortest Day at My House in Amsterdam (1970), conjunto de fotografías en las cuales el artista registró el transcurso del día durante el solsticio de invierno, o Brug Met Stip (1970), serie de postales comerciales a las que Dibbets añadió pegatinas circulares con la intención de comunicar su posición exacta en determinados momentos del día. Los mapas también le sirvieron para narrar pequeñas historias en la ciudad, historias de conexiones, desplazamientos y encuentros.

Para el Art \& Project Bulletin 15 (1969), Dibbets llevó a cabo numerosos envíos postales del boletín a diferentes personas que residían en Ámsterdam, el continente o distintos lugares del mundo, con instrucciones para que los receptores arrancaran parte del mismo y lo enviaran de nuevo a la galería por correo postal. El resultado de estos envíos recíprocos se condensó en una exposición que incluyó cuatro mapas intervenidos por el artista: Ámsterdam, Benelux ${ }^{4}$, Europa y el Mundo. En ellos, Dibbets destacó aquellos lugares desde los cuales se habían realizado los reenvíos a la galería, de modo que Ámsterdam es puesta en relación con diferentes visiones geográficas en un alejamiento progresivo. De cada una

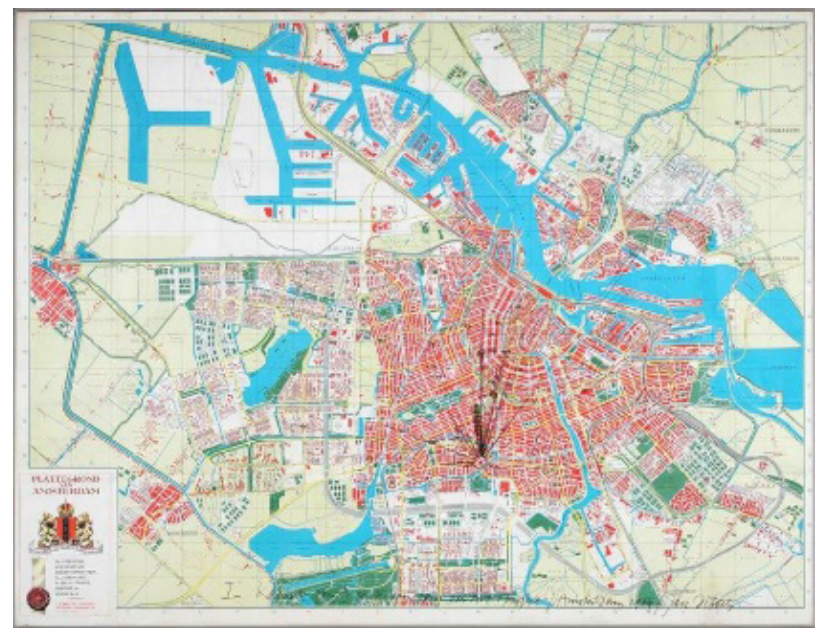

Imagen 6. Jan Dibbets. Project voor Art \& Project Bulletin 15, 1969.

de las direcciones postales parten líneas que vienen a converger en el espacio de la galería, generándose así una trama colectiva y multidireccional, un mapa de conexiones que une emisarios y receptores mientras la propia dirección del envío altera esos roles. El proyecto se exhibió en su primera exposición en Art \& Project en 1971.

Dibbets hará uso de la representación cartográfica en otros proyectos. El más poético de todos ellos, a medio camino entre el dibujo y la escultura, es Roodborst Territorium/Sculptuur (1969), en el cual el mapa registra una experiencia apenas perceptible. Se trata de un proyecto de cuatro meses de duración que el artista llevó a cabo en Vondelpark, con la intención de desplazar el territorio de un petirrojo: "después de aprender sobre las características intensamente territoriales de los petirrojos, Dibbets se interesó en alterar los hábitos del pájaro" (Cherix, 2009. Trad.

4 Acrónimo formado a partir de los nombres Bélgica, Nederland y Luxemburgo. El término designa la unión económica y aduanera de estos tres países, así como la región comprendida por ellos. 
propia). De alguna manera, la cartografía resultante representa también relaciones e intercambios mutuos, pero en esta ocasión entre el artista y el pájaro. Junto a algunas fotografías y breves escritos, el mapa registra y cuenta la historia del desplazamiento del petirrojo, una pequeña proeza que Dibbets logró instalando postes de madera, puntos de referencia geográfica y territorial para el pájaro, que fue gradualmente desplazado durante los meses en que se prolongó la realización del proyecto. Es resultado es un mapa que cuenta lo infraordinario, algo tan insignificante en el tiempo y el espacio de una ciudad como es el territorio de un ave en un parque.

\section{APUNTES HACIA UNA CONCLUSIÓN}

Tanto Jan Dibbets como Stanley Brouwn y Sol LeWitt recurrieron al trabajo con mapas para contar pequeñas historias de la ciudad y generar representaciones parciales, subjetivas o fragmentarias de la misma. Estas historias tratan de lugares habitados y recorridos, encuentros en el espacio público, conexiones postales entre Ámsterdam y el resto del mundo o desplazamientos mínimos. En las representaciones cartográficas resultantes, Ámsterdam se nos muestra múltiple, compleja y poliédrica.

La representación de la ciudad es siempre mutable y las prácticas artísticas pueden hacer uso del mapa para promover nuevos imaginarios de la urbe contemporánea, para contar aquello que el mapa oficial no cuenta y que el mapa turístico olvida. No es, por tanto, en la representación del imperio ni del país que narraban Carroll o Borges, sino en estas pequeñas tentativas y escalas donde el mapa desborda el límite para reencontrar su potencia representativa y su capacidad narrativa. En los ejemplos citados a lo largo de este escrito, la cartografía no es un instrumento que trata de sustituir representacionalmente a la realidad, sino de capturar o dar cuenta de la experiencia compleja de la misma. El mapa se con- vierte, así, en un dispositivo poético a disposición de las prácticas artísticas.

\section{REFERENCIAS:}

Borges, J. L. (1974). Del rigor en la Ciencia. En: Borges, J. L. Obras Completas 1923-1972 (p.847). Buenos Aires: Emecé Editores.

Brouwn, S. (1969). Art \& Project Bulletin \#11. Amsterdam: Art \& Project.

Brouwn, S. (2006). This way Brouwn. 25-2-61/26-261, Zeichnungen 1. En: Osborne, P. Arte conceptual (p.130). Barcelona: Phaidon.

Carroll, L. (2015). Sylvie and Bruno Concluded. Project Gutemberg Ebook. Recuperado el 15 de diciembre de 2017 de http://www.gutenberg.org/ebooks/620

Cherrix, C. (2009). In \& Out of Amsterdam: Travels in Conceptual Art, 1960-1976. Recuperado el 17 de noviembre de 2017 de: https://www.moma.org/ interactives/exhibitions/2009/inandout/

Cherix, C. (2009). In \& Out of Amsterdam: Travels in Conceptual Art 1960-1976. Catálogo de la exposición. New York: The Museum of Modern Art.

Debord, G. (1977). Teoría de la deriva. En: VV.AA. La creación abierta y sus enemigos. Textos situacionistas de arte y urbanismo (pp.61-69). Madrid: La Piqueta.

Fabrizi, M. B. (2015). Tearing, Cutting and Folding: Early Map Works by Sol LeWitt (1967-1979). En: Socks Studio. Recuperado el 7 de junio de 2017 de: http:// socks-studio.com/2015/01/27/tearing-cutting-andfolding-early-map-works-by-sol-lewitt-1967-1979/

Speranza, G. (2012). Atlas portátil de América Latina. Barcelona: Anagrama.

Van den Boogaard, O. (2014). In search of Stanley Brouwn. Frieze. Recuperado el 15 de noviembre de 2017 de: https://frieze.com/article/ 